\title{
Erratum to: Off-limb (Spicule) DEM Distribution from SoHO/SUMER Observations
}

\author{
K. Vanninathan · M.S. Madjarska $\cdot$ E. Scullion • \\ J.G. Doyle
}

Published online: 30 September 2014

(C) Springer Science+Business Media Dordrecht 2014

\section{Erratum to: Solar Phys. (2012) 280:425-434 \\ DOI 10.1007/s11207-012-9986-8}

A mistake in our program has lead to the erroneous calculation of the spicule DEM. The figures and calculations given in the paper have changed. The corrected versions are presented here. We apologize for this late clarification.

Figure 2 from the paper should be changed to the one below.

The values for radiance given in Table 1 have been corrected.

The modified version of Figure 4 is given below.

The calculations we did in Section 4 are modified and should read as:

From the graphs in Figure 4 we were able to numerically integrate the values to obtain what percent of plasma seen in the $171 \AA$ filter is above million degrees. We find that in the case of spicule or prominence observations the contribution is only $1.3 \%$ and $2.5 \%$, respectively, whereas for the quiet Sun it is a little over $23 \%$.

However, our main conclusion still remains the same. During spicule observations the emission in the Fe IX $171 \AA$ filter comes from plasma at transition region temperatures rather than coronal.

The online version of the original article can be found under doi:10.1007/s11207-012-9986-8.

K. Vanninathan $(\varangle) \cdot$ M.S. Madjarska · J.G. Doyle

Armagh Observatory, College Hill, Armagh BT61 9DG, Northern Ireland, UK

e-mail: kva@arm.ac.uk

K. Vanninathan

School of Mathematics and Physics, Queen's University Belfast, Belfast BT7 1NN, Northern Ireland, UK

E. Scullion

Institute of Theoretical Astrophysics, University of Oslo, P.O. Box 1029, Blindern, 0315 Oslo, Norway 
Figure 2 The DEM for off-limb data (where spicules are most easily identified) is calculated for different abundances as indicated in the legend using CHIANTI 7.1 .

Table 1 Details of the SUMER spectral lines used for the construction of the DEM.

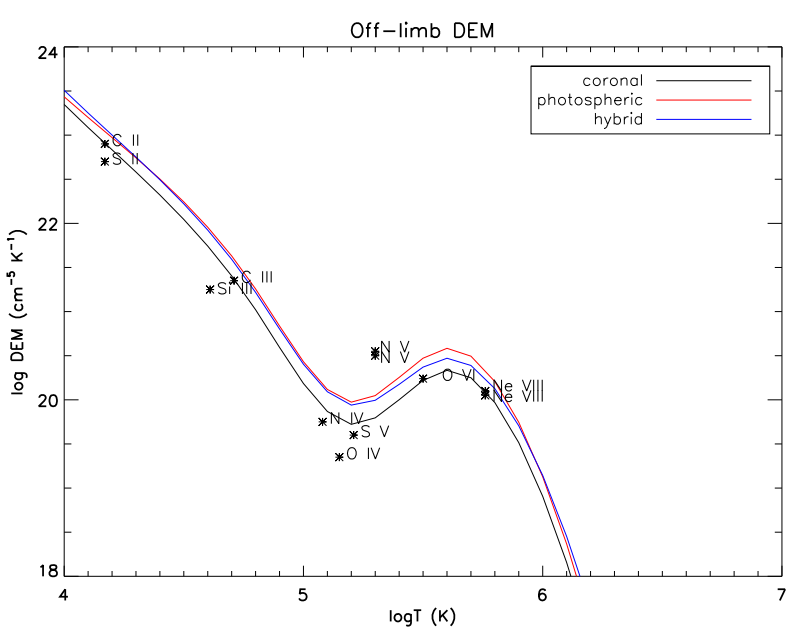

\begin{tabular}{|c|c|c|c|}
\hline Wavelength $(\AA)$ & Ion & $\log T_{\max }(\mathrm{K})$ & $\begin{array}{l}\text { Radiance } \\
\left(\operatorname{ergs~cm} \mathrm{cm}^{-2} \mathrm{~s}^{-1} \mathrm{sr}^{-1}\right)\end{array}$ \\
\hline 765.150 & $\mathrm{~N}$ IV & 5.1 & 12.5 \\
\hline 770.420 & $\mathrm{Ne}$ VIII & 5.8 & 16.9 \\
\hline 780.300 & $\mathrm{Ne}$ VIII & 5.8 & 8.5 \\
\hline 786.470 & S V & 5.2 & 4.9 \\
\hline 787.720 & O IV & 5.2 & 9.3 \\
\hline 977.030 & C III & 4.8 & 314.9 \\
\hline 1031.93 & $\mathrm{OVI}$ & 5.5 & 164.1 \\
\hline 1238.82 & $\mathrm{~N} \mathrm{~V}$ & 5.3 & 50.6 \\
\hline 1242.80 & $\mathrm{~N} v$ & 5.3 & 24.5 \\
\hline 1253.80 & S II & 4.2 & 1.1 \\
\hline 1298.96 & Si III & 4.7 & 4.0 \\
\hline 1334.53 & C II & 4.4 & 90.5 \\
\hline
\end{tabular}



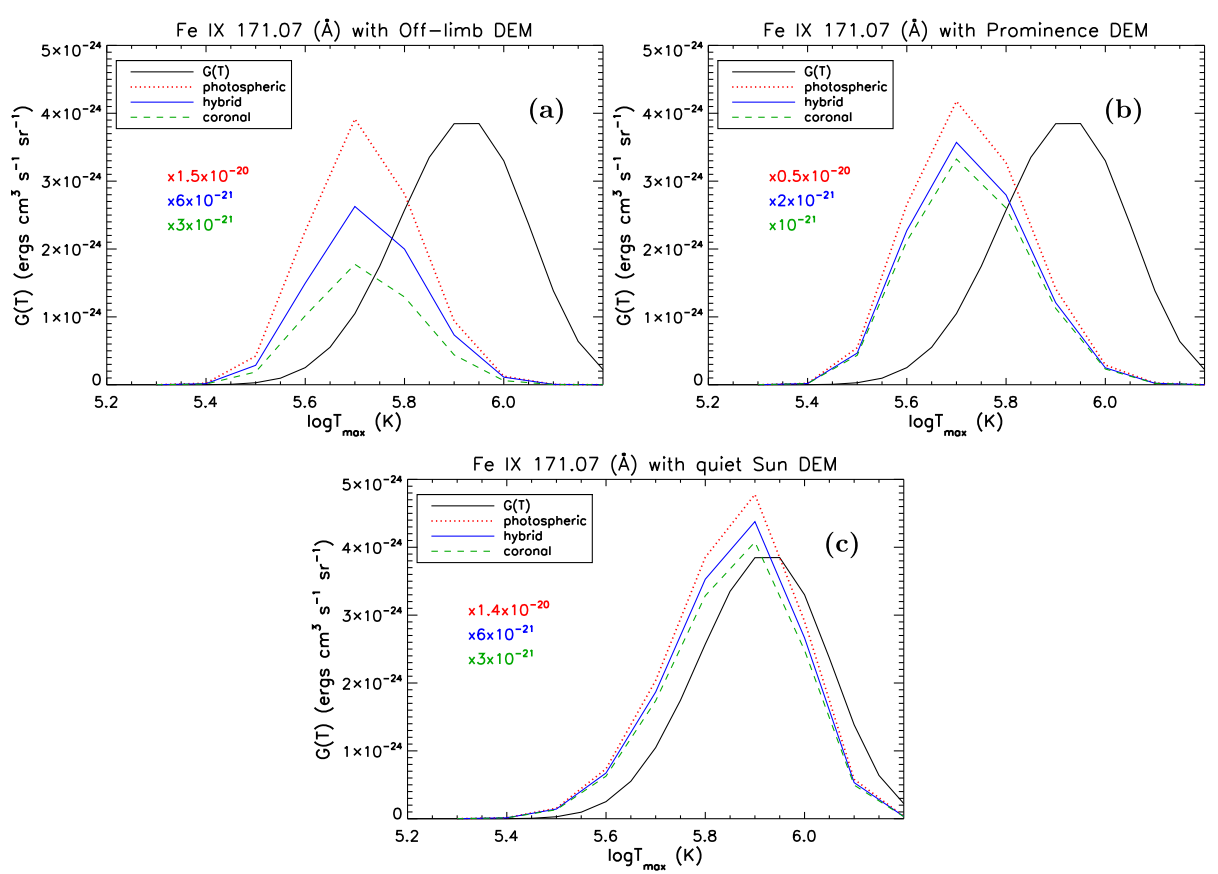

Figure 4 The contribution function $G(N, T)$ for Fe IX $171.07 \AA$ (bold, solid line) is plotted along with the normalised product $G(N, T) \times \operatorname{DEM}(T)$ for three different DEMs (a) off-limb, (b) prominence and (c) quiet Sun and for different solar abundances as indicated in the legend. 